\title{
Particle acceleration at relativistic shock waves
}

\author{
Martin Lemoine ${ }^{1}$, Guy Pelletier ${ }^{2}$ \\ ${ }^{1}$ Institut d'Astrophysique de Paris, CNRS, UPMC, 98 bis boulevard Arago, 75014 Paris, France \\ 2 Institut de Planétologie et d'Astrophysique de Grenoble, CNRS, Université Joseph Fourier-II, 34 \\ rue de la Piscine, 38041 Grenoble, France
}

\begin{abstract}
Relativistic sources, e.g. gamma-ray bursts, pulsar wind nebulae and powerful active galactic nuclei produce relativistic outflows that lead to the formation of collisionless shock waves, where particle acceleration is thought to take place. Our understanding of relativistic shock acceleration has improved in the past decade, thanks to the combination of analytical studies and high level numerical simulations. In ultra-relativistic shocks, particle acceleration is made difficult by the generically transverse magnetic field and large advection speed of the shocked plasma. Fast growing microturbulence is thus needed to make the Fermi process operative. It is thought, and numerical simulations support that view, that the penetration of supra-thermal particles in the shock precursor generates a magnetic turbulence which in turn produces the scattering process needed for particle acceleration through the Fermi mechanism. Through the comparison of the growth timescale of the microinstabilities in the shock precursor and the precursor crossing timescale, it is possible to delimit in terms of magnetization and shock Lorentz factor the region in which micro-turbulence may be excited, hence whether and how Fermi acceleration is triggered. These findings are summarized here and astrophysical consequences are drawn.
\end{abstract}

Keywords: collisionless shock waves; relativistic shock waves; particle acceleration; gamma-ray bursts.

\section{Introduction}

Broadband spectra of powerful astrophysical objects, from the radio range, through X-rays, gammarays up to $\mathrm{TeV}$ energies, unerringly reveal non-thermal powerlaws that are interpreted as the secondary radiation of accelerated charged particles. Relativistic collisionless shock waves are most commonly discussed as natural sites for particle acceleration, for good and various reasons. First and foremost, these shock waves are the natural consequences of the relativistic outflows associated with relativistic sources such as microquasars, active galactic nuclei, gamma-ray bursts, pulsar wind nebulae, which all harbour a central object producing a relativistic wind. As viewed in the frame in which the shock front remains at rest, the incoming kinetic energy flux $\gamma_{\mathrm{sh}}^{2} n_{\mathrm{u}} m c^{2}$ of a cold upstream population $-\gamma_{\mathrm{sh}}$ denotes the Lorentz factor of the shock wave, $n_{\mathrm{u}}$ the proper density of the upstream 
(unshocked) plasma - flows through the shock and gets converted into thermal disordered energy with mean energy per particle $\sim \gamma_{\mathrm{sh}} m c^{2}$. Provided a reasonable fraction $(\sim 1-10 \%)$ of this energy flux is converted into a non-thermal radiation powerlaw, one is able to explain the large amount of radiation produced in these sources. In the particular case of gamma-ray bursts, there exists a substantial body of evidence arguing in favor of shock acceleration of electrons at the external shock front of the wind as it impinges on the circumburst medium in relation to the observed afterglow emission (e.g. Piran 2005 for a review). There exists also overwhelming evidence for particle acceleration at the non-relativistic counterparts of such collisionless shock waves, either in the interplanetary medium or in supernovae remnants. Finally, from a theorist perspective, understanding how particle acceleration takes place around a shock wave appears as a well posed problem, the physics of which appears controlled by a limited set of parameters, most notably the Lorentz factor of the shock wave $\gamma_{\mathrm{sh}}$ and the magnetization level $\sigma^{1}$

The Fermi process of shock acceleration is well known, at least in the non-relativistic regime and in the test particle limit in which one neglects the influence of the accelerated particles on the shock environment. The incoming plasma flow (as viewed from the shock front rest frame) is supposed to carry a frozen in turbulent magnetic field; high energy particles scatter off magnetic perturbations with a mean free path much larger than the shock thickness, so that they can cross the shock front back and forth; these particles undergo elastic interactions with magnetic disturbances in the proper frame of these latter, however upstream magnetic perturbations move faster than downstream ones, therefore a particle that undergoes a Fermi cycle - i.e. a cycle upstream-downstream-upstream or downstream-upstream-downstream - picks up energy in a systematic way from the convective electric fields. Even in this test particle limit, relativistic shock acceleration reveals substantial differences with its non-relativistic counterpart, as revealed by Achterberg et al. (2001). Yet as we have learned in the past ten years, the test particle limit obliviates effects that are crucial in the relativistic regime. What is unusual in astrophysics, it becomes mandatory to consider the microphysics of the acceleration process, in particular the intimate non-linear relationship between the accelerated particles and the shock structure, as will be stressed in the following.

This short review is organized as follows. Section 2 discusses first the test particle picture of relativistic shock acceleration and its limitation; a more modern view of this process is then presented. Section 3 discusses some applications to high energy astrophysics, regarding notably the acceleration in gamma-ray bursts, pulsar wind nebulae and active galactic nuclei, as well as the possibility of accelerating particles to ultra-high energies in relativistic shocks.

\footnotetext{
${ }^{1}$ For definiteness, we recall that at normal incidence, a strong weakly magnetized shock moves at velocity $c / 3$ away from the shocked (downstream) plasma, which itself moves toward the unshocked (upstream) plasma with bulk Lorentz factor $\gamma_{\mathrm{sh}} / \sqrt{2}$ (Blandford \& McKee 1976). The magnetization level is defined as the ratio of the incoming magnetic energy flux to the incoming kinetic energy flux, $\sigma=B_{\mathrm{u}}^{2} /\left(4 \pi n_{\mathrm{u}} m_{p} c^{2}\right)$ as measured in terms of upstream quantities, i.e. $\sigma=\left(v_{\mathrm{A}} / c\right)^{2}$ in terms of the upstream Alfvén speed (for $\sigma \ll 1$ ).
} 


\section{Relativistic shock acceleration}

\subsection{A simplified view: the test particle limit}

One of the most dramatic success of Fermi acceleration at shock waves is to predict the formation of a powerlaw of accelerated particles with differential energy spectrum index $s \approx 2$, i.e. approximately constant energy per logarithmic energy bin, thanks to the competition between energy gain and escape, in good qualitative agreement with a variety of observed powerlaws. In the non-relativistic limit, this competition leads to the formula for diffusive shock acceleration (Bell 1978): $s=1-$ $\ln \left(P_{\text {ret }}\right) / \ln (1+\Delta E / E)$, where $P_{\text {ret }}$ represents the probability of remaining at the acceleration site after a Fermi cycle around the shock front ${ }^{2}$ and $\Delta E / E$ the relative energy gain per cycle. A relativistic variant of this formula has been proposed by Vietri (2003).

As noted by Gallant \& Achterberg (1999), Achterberg et al. (2001), relativistic shock acceleration differs substantially from its non-relativistic counterpart, because the shock wave moves with a velocity $\sim c$ close to that of the accelerated particle. One of the most dramatic consequences is to limit the energy gain per cycle to a modest $\Delta E / E \sim \mathcal{O}(1)$. Together with an escape probability per cycle $1-P_{\text {ret }} \sim 0.4$, this leads to the formation of a powerlaw with spectral index $s \sim 2.2-2.3$, at least if the scattering process is isotropic. This value has been derived through semi-analytical techniques (Kirk et al. 2000, Achterberg et al. 2001), Monte Carlo simulations (Bednarz \& Ostrowski 1998, Ellison \& Double 2002, Lemoine \& Pelletier 2003) and analytical estimates (Keshet \& Waxman 2005) but one must emphasize that this value assumes efficient acceleration (to be discussed further below) and isotropic scattering. The latter might turn out to be a poor assumption, all the more so as the shock normal sets a privileged direction, e.g. shock compression compresses the magnetized turbulence along this direction. Accounting for such anisotropy, one finds different spectra (Keshet 2006, Lemoine \& Revenu 2006).

More importantly, if one adheres to the test particle limit, one is led to the conclusion that Fermi acceleration fails in the highly relativistic regime unless very special circumstances are met. This can be argued as follows but first of all, let us stress that relativistic shock waves are quite generically superluminal (Begelman \& Kirk 1990): this occurs when the angle $\Theta_{B}$ between the magnetic field in the upstream rest frame and the shock normal verifies $\Theta_{B} \gtrsim 1 / \gamma_{\mathrm{sh}}$ if $\gamma_{\mathrm{sh}} \gg 1$. Superluminal regime means that the intersection point of a magnetic field line with the shock surface moves faster than light across this surface. Hence, if a particle were tied to a field line, it would not be able to cross repeatedly back and forth the shock front. To put it otherwise, the magnetic field can be considered as almost perpendicular in the front frame, since its transverse component is amplified by a factor $\sim \gamma_{\mathrm{sh}}$ relatively to the longitudinal component (i.e. along the shock normal). Particles must therefore diffuse across the field lines in order to complete Fermi cycles.

In principle, cross-field diffusion is possible in large scale turbulence - large means here that most of the power lies at a scale that is much larger than the typical Larmor radius of the accelerated particle - and it had been hoped that such diffusion would allow particle acceleration at relativistic shock waves. But an usual turbulent MHD state with a large scale coherence length behaves like an ordered magnetic field for the suprathermal particles, because their penetration length upstream

\footnotetext{
${ }^{2}$ for a steady planar shock, escape occurs through advection towards downstream with probability $1-P_{\text {ret }}$, as the shock front moves away from downstream but towards upstream
} 
remains much shorter than the coherence length of the turbulence and the expected duration of the cycle is much smaller than the eddy turn over time. It can thus be shown analytically that, in such turbulence, a particle cannot execute more than one and a half Fermi cycle before being dragged away far downstream (Lemoine et al. 2006). As the particle gets advected away, it takes a timescale $\sim D_{\|} / c^{2}$ (with $D_{\|}$the parallel diffusion coefficient in the turbulence) for the particle pitch angle to start diffusing; however, by that time, the particle lies much further away from the shock front than a perpendicular diffusion length, since $D_{\|} \gg D_{\perp}$ and the shock front moves away at large velocity $\simeq c / 3$. The particle never catches up with the shock front, as it moves across the field lines at a much smaller effective velocity. Such inhibition of Fermi cycles in the relativistic limit has been observed in test particle Monte Carlo simulations by Niemiec \& Ostrowski (2006) and Niemiec et al. (2006).

When does relativistic Fermi acceleration then take place? In the simplest case, one can argue that acceleration is to occur provided intense short scale turbulence has been excited, on scales $\lambda_{B} \lesssim r_{\mathrm{L}}$ with intensity $\delta B / B \gg 1$ (Pelletier et al. 2009). Under the conditions discussed in this work, the micro-turbulence unlocks the particles off the field lines that would otherwise carry them away from the shock front. When and where such conditions are met nicely explain the results of Niemiec et al. (2006). More sophisticated scenarios for acceleration include e.g., radiative interactions during the Fermi cycles (Derishev et al. 2003) or magnetic dissipation in the shock transition (Lyubarsky 2003); they will be addressed further below.

That short scale turbulence would be excited in the vicinity of a collisionless shock should not be regarded as a surprise. In the absence of collisions, small scale electromagnetic fields are to play the agents that slow down the flow and dissipate the incoming kinetic energy. The accelerated particles, as forerunners of the shock are susceptible of exiciting such small scale turbulence. This is where one has to abandon the test particle description and consider the backreaction of these accelerated particles on the shock structure.

There exist interesting connections with observations in this context. In particular, the standard modeling of gamma-ray burst afterglow spectra points to a much higher level of magnetized turbulence in the shocked region than in the interstellar medium, by some 5 orders of magnitude in magnetic field intensity (Gruzinov \& Waxman 1999). This implies that the turbulence is self-generated in the blast. A leading candidate for the source of such turbulence is the Weibel (filamentation) instability in the shock precursor, between the accelerated particle population and the incoming (upstream) plasma population (Medvedev \& Loeb 1999), which produces filaments on a plasma scale $\sim c / \omega_{\mathrm{pi}}$ (with $\omega_{\mathrm{pi}}$ the upstream ion plasma frequency). If the gamma-ray burst explodes in the interstellar medium, $c / \omega_{\mathrm{pi}} \sim 10^{7} \mathrm{~cm}$ is much smaller than the typical Larmor radius of suprathermal particles $r_{\mathrm{L}, 0} \sim 10^{12} \mathrm{~cm}$ (as measured in the downstream frame, in the shock compressed interstellar field).

Moreover, by comparing the acceleration and the cooling timescale, Li \& Waxman (2006) [see also Li \& Zhao (2011)] have argued that the generation of the early X-ray afterglow requires a much larger magnetic field in the shock precursor than in the interstellar medium. Unless the circumburst medium is substantially magnetized - a possibility considered further below - turbulence must have been excited in the precursor, and if so, on very short length scales. Indeed, the precursor itself is at most of extent $r_{\mathrm{L}, 0} / \gamma_{\mathrm{sh}}^{3} \sim 10^{10} \mathrm{~cm}$, as measured in the upstream rest frame, with $r_{\mathrm{L}, 0}$ denoting 
the Larmor radius of suprathermal particles in the background magnetic field, the numerical value corresponding to $B_{\mathrm{u}}=1 \mu \mathrm{G}$ and $\gamma_{\mathrm{sh}}=300$. This scaling with $\gamma_{\mathrm{sh}}$ arises because the distance between a suprathermal particle and the shock front increases as $\left(1-\beta_{\mathrm{sh}}\right) c \Delta t \simeq c \Delta t /\left(2 \gamma_{\mathrm{sh}}^{2}\right)$ with time $\Delta t$ since shock crossing, and because the typical residence time spent upstream (i.e. before return to the shock front) $\Delta t \sim r_{\mathrm{L}, 0} / \gamma_{\mathrm{sh}}$ (Achterberg et al. 2001, Milosavljevic \& Nakar 2006, Pelletier et al. 2009).

\subsection{Microphysics of shock acceleration}

The above teaches us that the generation of turbulence in the shock environment, the process of particle acceleration and the structure of the shock itself form an inseparable tryptich, which must be considered as a whole. As studies of non-relativistic magnetospheric shocks have taught us, the reflection of a fraction of the incoming (upstream) population on the shock front constitutes an essential ingredient of the formation of the collisionless shock (e.g. Leroy 1983). In the shock precursor, where both the reflected particles and the incoming plasma meet, dissipation and the build-up of an electromagnetic barrier are initiated through micro-instabilities.

The investigation of ultra-relativistic collisionless shocks started at the turn of 1990 with J. Arons and co-workers, in the case of high magnetization, say $\sigma>0.03$ (Hoshino \& Arons 1991, Hoshino et al. 1992, Gallant et al. 1992). The results revealed the interesting physics of such shocks: the loop of particles that are reflected by an intense magnetic barrier triggers a synchrotron maser instability that in turn radiates a coherent electromagnetic wave towards upstream as well as electromagnetic waves towards downstream that are absorbed, thereby heating the particle population. If the incoming plasma is composed of electrons and protons, the upstream coherent waves exerts a ponderomotive force on the electrons, which leads to wakefield heating/acceleration. Whether heating or powerlaw acceleration occurs is presently debated on the basis of recent particle-in-cell (PIC) simulations, see Hoshino (2008) and Sironi \& Spitkovsky (2011a).

The physics of the shock wave differs at lower magnetization. The breakthrough PIC simulations of an unmagnetized shock (no mean field, i.e. $\sigma=0$ ) in a pair plasma with $\gamma_{\mathrm{sh}} \simeq 20$, conducted by Spitkovsky (2008) has validated the paradigm that combines the three fundamental processes: the formation of a collisionless relativistic shock front with reflected particles, the generation of magnetic turbulence and the generation of a power law distribution through Fermi process. Reflected particles run ahead of the shock, initiate the growth of magnetic micro-turbulence through streaming type instabilities, most notably the Weibel (filamentation) branch; and, self-consistently, the spatial growth of the turbulence produces a partial reflection of the incoming population, which sustains the dynamics of the shock front, the generation of turbulence, etc. The simulation also indicate the formation of a powerlaw of accelerated particles, thanks to the scattering on the turbulence that these particles self-excite.

Particle-in-cell simulations have by now become an invaluable tool for addressing the complex non-linear microphysics of these relativistic collisionless shock waves. Recent simulations have confirmed the onset of Fermi acceleration in unmagnetized pair and electron-proton plasmas (Martins et al. 2009) and indicated the absence of powerlaw acceleration in (superluminal) highly magnetized shock waves with $\sigma$ close to unity (Sironi \& Spitkovsky 2009, 2011a).

As powerful as they are, current PIC simulations cannot yet probe the temporal or spatial scales 
that are associated to observed high energy phenomena. For instance, the long simulations of Sironi \& Spitkvosky $\left(2009,2011 \mathrm{a}\right.$ ) extend to $\sim 10^{3} \omega_{\mathrm{pi}}^{-1}$ (downstream rest frame), i.e. a fraction of a second for a typical interstellar environment, which remains small compared to the comoving dynamical timescale of a gamma-ray burst external shock wave, $R /\left(\gamma_{\mathrm{sh}} c\right) \sim 10^{4}$ sec. Interestingly, the longer simulations of Keshet et al. (2009), $\sim 10^{4} \omega_{\text {pi }}^{-1}$, do not reveal a stationary shock structure but point towards a magnetized turbulence that increases in scale and strength with time. It is tempting to relate, as these authors do, such behavior with the acceleration of particles to progressively higher energies.

The above remarks and findings lead to the following picture for relativistic Fermi acceleration. The Fermi process takes place when micro-turbulence can be generated with $\delta B \gg B$ and $\lambda_{B} \ll r_{\mathrm{L}}$, as discussed previously. Otherwise, the superluminal configuration of the magnetized shock wave, even weakly magnetized, halts prematurely the Fermi cycles. Assuming that the micro-turbulence has been generated and survives behind the shock front, one can check that the first generations of Fermi accelerated particles scatter on this turbulence. To see this, write $\epsilon_{B}$ the fraction of shock dissipated energy density $2 \gamma_{\mathrm{sh}}^{2} n_{\mathrm{u}} m c^{2}$ (with $m=m_{p}$ for an $e-p$ shock, $m=m_{e}$ for a pair shock) that has been stored in magnetized microturbulence on typical scale $\lambda_{B}$. Particle-in-cell simulations indicate $\epsilon_{B} \sim 10^{-2}$ and $\lambda_{B} \sim c / \omega_{\mathrm{pi}}$ for weakly magnetized shocks. Then the ratio $r_{\mathrm{L}} / \lambda_{B} \sim \epsilon_{B}^{-1 / 2} \gamma / \gamma_{\min }$, with $\gamma$ the Lorentz factor of the particle and $\gamma_{\min }$ the Lorentz factor of shock heated particles; for electrons, $\gamma_{\min } \sim \gamma_{\mathrm{sh}} m_{p} / m_{e}$ if equipartition with the protons is reached, as PIC simulations indicate (Sironi \& Spitkovsky 2011a). One then obtains $r_{\mathrm{L}} \gtrsim \lambda_{B}$, meaning that the suprathermal particles effectively probe the immediate vicinity of the shock. How the turbulence evolves away from the shock remains an important open question (Chang et al. 2008, Keshet et al. 2009), which may influence the physics of acceleration to higher energies (Katz et al. 2007).

But, to accelerate particles to high energies, one first need to ignite the Fermi process and this can be understood as follows. A suprathermal particle carries a typical energy $\gamma_{\mathrm{sh}}^{2} m c^{2}$, as measured in the upstream rest frame; let $\xi_{\text {cr }}$ denote the fraction of shock dissipated energy carried by the suprathermal population. For purely kinematic reasons, these particles make up a sharp beam in phase space with small angular dispersion $\lesssim 1 / \gamma_{\mathrm{sh}}$ along the shock normal; indeed, particles with angle $\gtrsim 1 / \gamma_{\text {sh }}$ travel along the shock normal at a velocity $\lesssim \beta_{\text {sh }}$ hence they cannot outrun the shock (Gallant \& Achterberg 1999). This beam of particles carry a plasma frequency $\omega_{\mathrm{pb}} \simeq$ $\xi_{\text {cr }}^{1 / 2}\left(m_{e} / m_{p}\right)^{1 / 2} \omega_{\text {pe }}$, much smaller than the background plasma frequency, even though the beam density is much larger, $n_{\mathrm{b}} \simeq \gamma_{\mathrm{sh}}^{2} n_{\mathrm{u}}$. Through its mixing with the background plasma particles, this beam induces streaming instabilities. Various instabilities have been considered in the literature, see Bret (2009), Lemoine \& Pelletier (2010), and Bret et al. (2010) for a compilation of fast growing modes, but the fastest appear to be the well studied filamentation instability (Medvedev \& Loeb 1999, Wiersma \& Achterberg 2004, Lyubarsky \& Eichler 2006, Achterberg \& Wiersma 2007, Achterberg et al. 2007, Lemoine \& Pelletier 2010, 2011a, Rabinak et al. 2010) and the two stream instability in its oblique version (Bret et al. 2005, Lemoine \& Pelletier 2010, 2011a). In a weakly magnetized $e-p$ shock, the electrons can be efficiently preheated to near equipartition in the shock precursor (Lemoine \& Pelletier 2011a), in which case the filamentation instability becomes the fast growing mode.

The unstable modes grow only if their growth timescale $\tau_{\text {inst. }}$ is shorter than the time it takes 
for the precursor to overtake the plasma element. In detail, this means $\tau_{\text {inst. }}<r_{\mathrm{L}, 0} /\left(\gamma_{\mathrm{sh}}^{3} c\right)$, given the precursor length scale discussed earlier. For the filamentation instability, this condition can be rewritten (see Lemoine \& Pelletier 2010):

$$
\xi_{\mathrm{cr}}^{-1} \gamma_{\mathrm{sh}}^{2} \sigma<1
$$

The higher the magnetization, or the larger the shock Lorentz factor, the shorter the precursor, hence the lesser the amount of time for microturbulence to grow.

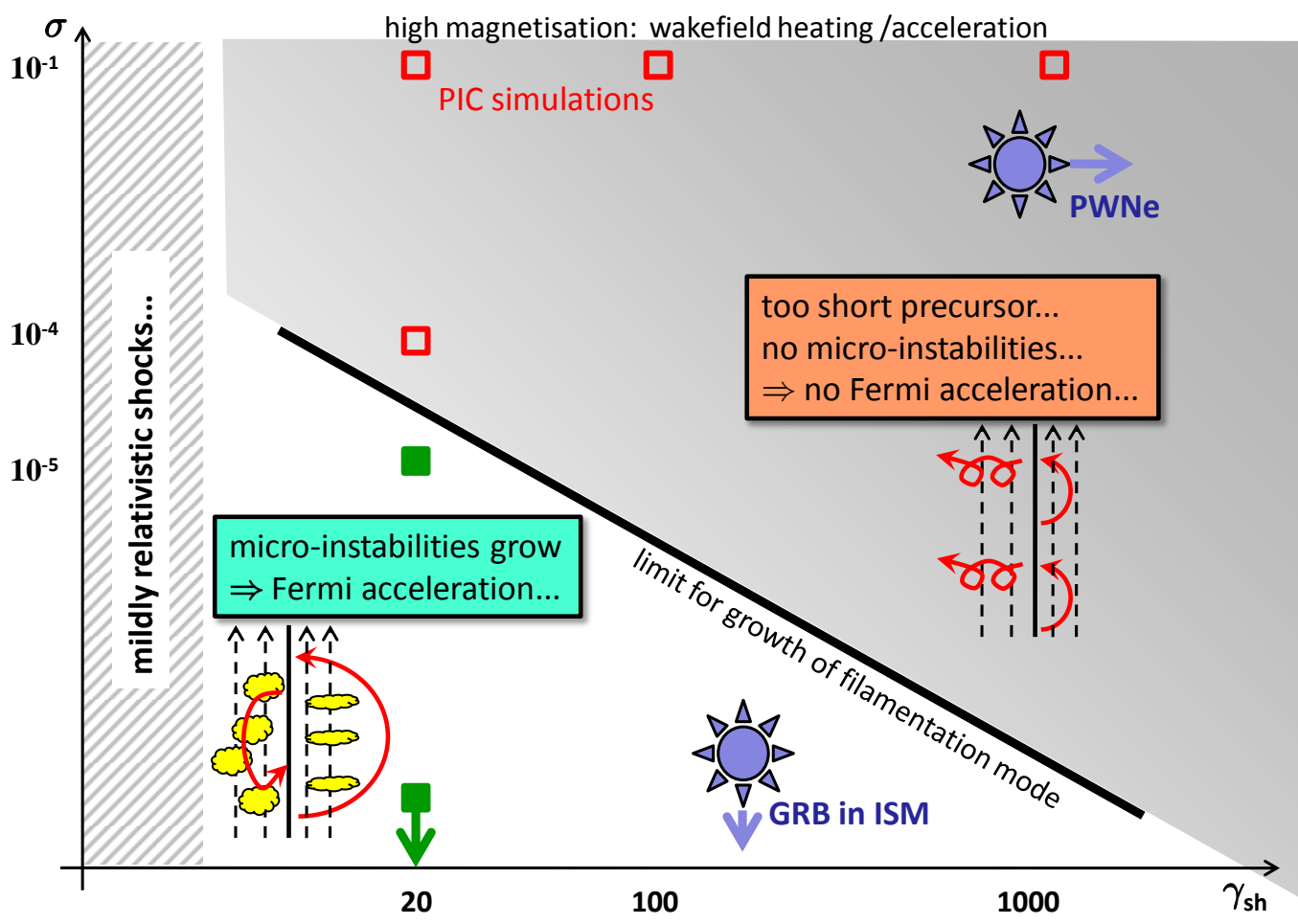

Figure 1: Parameter space for relativistic shocks with shock Lorentz factor $\gamma_{\mathrm{sh}}$ in abscissae and magnetization of the incoming plasma $\sigma$ in ordinates. In the gray region, the precursor is too short to allow the growth of micro-instabilities by suprathermal particles, hence Fermi acceleration cannot take place (under the assumptions discussed in the text). The squares indicate the results of recent PIC simulations (Sironi \& Spitkovsky 2011a), which validate where applicable, this model: empty squares indicate no evidence for particle acceleration while filled squares mean that powerlaw Fermi type acceleration has been observed. The region at low $\gamma_{\mathrm{sh}}$ corresponding to mildly relativistic shocks is yet unexplored. See Lemoine \& Pelletier (2010) for a more detailed version of this figure.

This leads to Fig. 1 which delimits the region of parameter space $\gamma_{\mathrm{sh}}$ (abscissae) $-\sigma$ (ordinates) 
in which the above condition is satisfied. This figure must be understood as a qualitative representation and more quantitative details can be found in Lemoine \& Pelletier (2010). The results of recent PIC simulations (Sironi \& Spitkovsky 2011a) for $e-p$ shocks with $\gamma_{\mathrm{sh}}=20$ are represented as open (red) squares when no evidence for particle acceleration has been observed in these simulations, or as filled (green) squares if particle acceleration has been observed. For this restricted set of simulations, the agreeement is therefore highly satisfactory. In Fig. 1 the limit has been taken at $\xi_{\mathrm{cr}}^{-1} \gamma_{\mathrm{sh}}^{2} \sigma=0.3$, corresponding to a few efolds of growth of the turbulence on a precursor crossing timescale. This figure also gives typical positions in this diagram for gamma-ray burst external shock waves propagating in the interstellar medium (annotated "GRB in ISM") and pulsar wind nebulae ("PWNe"). These specific objects will be discussed in more detail in the following.

Figure 1 omits the growth of Whistler waves in the precursor. As discussed in Lemoine \& Pelletier (2010), such waves can grow in the cold plasma limit for magnetization levels as high as $\xi_{\mathrm{cr}} \gamma_{\mathrm{sh}}^{-3} m_{p} / m_{e}$, hence $\sigma \sim 10^{-2}$ for $\gamma_{\mathrm{sh}} \sim 20, \xi_{\mathrm{cr}} \sim 0.1$. However, the Whistler inertial range disappears progressively as electrons are heated to relativistic temperatures, and the growth rate also decreases. Sources of electron heating are discussed in Lemoine \& Pelletier (2011a); they include, in particular, the oblique two stream instability and the Buneman instability at the tip of the precursor.

To summarize, relativistic Fermi acceleration requires a weakly magnetized upstream medium, all the more so at large Lorentz factors. Accelerated particles scatter and possibly cool in the turbulence that they themselves excite. Yet, many questions remain open. To quote but a few: the fate of the turbulence in the post-shock plasma, which controls the fate of acceleration to higher energies (as higher energy particles probe longer distances in the post-shock plasma), the dynamical evolution of the shock structure with time, and the physics of acceleration in the mildly relativistic $\left(\gamma_{\mathrm{sh}} \sim 1-5\right)$ regime, shown in cross-hatched in Fig. 1 .

The above discussion rests on a few specific assumptions. In particular, one has neglected the radiative interactions of the accelerated particles in the course of the Fermi cycles, one has assumed the shock front to be steady, planar and one has also neglected dissipation of the magnetic field in the shock transition, or even an external source of turbulence. If either of these assumptions were violated, one could expect interesting developments with respect to particle acceleration, some of which are currently the focus of interest. Consider for instance the possibility of radiative interactions. As shown by Derishev et al. (2003), a charged particle can be converted through such an interaction into a neutral state which may then travel freely across the magnetic field. This neutral particle may later be converted back into the charged state well ahead of the shock front, thus opening Fermi cycles with widely different particle kinematics. For example, an electron could upscatter a photon, that would travel ahead of the shock, turn back through pair production into an electron/positron, that would turn around in the turbulence before encountering the shock front, while protons could be turned into neutrons through photopion interactions, the neutron decaying back into a proton ahead of the shock front... In this "converter" mechanism, the typical energy gain per cycle is much larger than unity, possibly as large as $\gamma_{\mathrm{sh}}^{2}$, due to the approximate isotropy of the suprathermal population. This may lead to distorted powerlaw spectra with interesting phenomenology.

If the magnetic field is dissipated in the shock transition through e.g., reconnection (Lyubarsky 
2003), or sourced externally in the shocked region, one may expect Fermi acceleration to become operative. Although, in the latter case, stringent conditions apply; in particular, the instability needs to grow faster behind the shock than a typical Larmor time of the suprathermal particles in the background magnetic field, otherwise these particles would not execute Fermi cycles.

\section{Consequences and applications}

This Section discusses some applications and consequences of the above microphysical view of relativistic shock acceleration for gamma-ray bursts, pulsar wind nebulae and powerful active galactic nuclei.

\subsection{Acceleration in astrophysical sources}

\subsubsection{Gamma-ray bursts}

Regarding gamma-ray bursts, there exist strong evidence - for a substantial fraction of observed long gamma-ray bursts - that the afterglow emission is associated with synchrotron emission of shock accelerated electrons with $\gamma_{\text {sh }} \sim 100$ at afterglow onset (e.g. Piran 2005 for a review). As the shock wave sweeps up matter, it decelerates and the external shock slowly transits towards the mildly relativistic then non-relativistic regimes.

If the magnetization is low and the external medium composed of electrons and protons, as one expects for an interstellar like circumburst medium $\left(\sigma \sim 10^{-9}\right.$ for $\left.B_{\mathrm{u}} \sim 3 \mu \mathrm{G}, n_{\mathrm{u}} \sim 1 \mathrm{~cm}^{-3}\right)$, micro-instabilities develop efficiently in the shock precursor and lead to efficient preheating of the electrons and efficient Fermi acceleration.

Since the scattering occurs in short scale turbulence, one may expect a radiative signature different from the standard synchrotron paradigm. The physics is here characterized by the socalled wiggler parameter $a \equiv e \delta B \lambda_{B} /\left(m_{e} c^{2}\right)$, which measures the capability of the magnetic force to deviate a relativistic electron of Lorentz factor $\gamma$ by an angle $1 / \gamma$ during the crossing of a coherence length (e.g. Medvedev 2000, Fleishman \& Toptygin 2007, Kirk \& Reville 2010). When $a>1$ the magnetic field produces a single deviation of the electron in the emission cone of half angle $1 / \gamma$, whereas when $a<1$ the electron can undergo several wiggles in the emission cone. For a relativistic shock, $a \sim \xi_{B}^{1 / 2} \gamma_{\mathrm{sh}} m_{p} / m_{e} \gg 1$, hence the emission behaves as normal synchrotron radiation in a mean field at the peak frequency, except that there is no polarization. A modification of the spectral shape may however appear in the small frequency domain, see e.g. (Medvedev 2006, Fleishman \& Urtiev 2010, Reville \& Kirk 2010).

Quite remarkably, there exists an almost universal energy limit when the electron scatters and cools in intense small scale turbulence: by comparing the scattering timescale $t_{\text {scatt }} \sim \gamma^{2} m_{e}^{2} c /\left(e^{2} \delta B^{2} \lambda_{B}\right)$, which characterizes the acceleration timescale, with the synchrotron cooling timescale $t_{\mathrm{syn}}=6 \pi m_{e} c /\left(\delta B^{2} \sigma_{\mathrm{T}} \gamma\right)$, one can bound the maximal Lorentz factor by above as (see also Kirk \& Reville 2010):

$$
\gamma_{\max } \simeq\left(\frac{6 \pi e^{2} \lambda_{B}}{\sigma_{\mathrm{T}} m_{e} c^{2}}\right)^{1 / 3} \approx\left(\frac{m_{p} / m_{e}}{n_{\mathrm{u}} r_{e}^{3}}\right)^{1 / 6} \sim 10^{6} n_{\mathrm{u}, 0}^{-1 / 6}
$$


where the second equality on the r.h.s. assumes $\lambda_{B}=c / \omega_{\mathrm{pi}}$ and $r_{e}$ denotes the classical electron radius. The corresponding peak energy for the emitted photons reads

$$
\epsilon_{\gamma, \max }=\frac{9}{4 \pi} \epsilon_{B} \gamma_{\mathrm{sh}}^{2} \frac{m_{p} c^{2}}{\gamma_{\max } \alpha_{\mathrm{e} . \mathrm{m} .}} \approx 1 \mathrm{GeV} \epsilon_{B,-2}^{1 / 2} \gamma_{\mathrm{sh}, 2.5}^{2} n_{\mathrm{u}, 0}^{1 / 6}
$$

with the usual generic notation: $Q_{x} \equiv 10^{-x} Q$ in cgs. Thus a single synchrotron-like spectrum extending up to several $\mathrm{GeV}$, even possibly a few tens, can be expected. The performance of relativistic shocks for electron acceleration and radiation is thus noteworthy. As electrons cool faster than a hydrodynamical time in the early afterglow, the conversion factor to radiation (synchrotron + inverse Compton) is large $\sim \epsilon_{e} \sim 10 \%$. Let us note finally that the inferred spectral index is generally in satisfactory agreement with an expected $s \sim 2.2$ for relativistic shock acceleration with isotropic scattering.

One must also consider the possibility that the outflow impinges on a magnetized circumburst medium, either because the interstellar magnetic field is relatively strong (meaning $B_{\mathrm{u}} \gtrsim 1 \mathrm{mG}$ ) or because the explosion takes place in a magnetized stellar wind. Recall that Li \& Waxman (2006) find $B \gtrsim 300 \mu \mathrm{G}$ for the upstream magnetic field during the early X-ray afterglow, which either imply amplification/generation of the magnetic field by streaming instabilities, as discussed earlier, or that the pre-existing magnetic field already verifies that limit. At such high magnetization, Fermi acceleration may well be initially inhibited but set on at later stages when $\gamma_{\text {sh }}$ has dropped to sufficiently low values. Such a scenario has been investigated in Lemoine \& Pelletier (2011b), where it is found that: initially, one records in the X-ray range the synchrotron emission of the thermal shock heated electron population; but, as the peak frequency exits the X-ray band on subday scales, one observes a drop-out in X-ray due to the absence of Fermi powerlaws on timescales $\sim 100 \mathrm{~s}$; finally, at later times, e.g. $\gtrsim 10^{4} \mathrm{~s}$, Fermi acceleration becomes operative, powerlaws develop and electrons radiate in X-rays, so that one recovers the standard afterglow light curve. This signature is interesting because of its definite character but also because it may explain some of the peculiar X-ray light curves recently observed by the Swift satellite.

The above considerations apply to the ultra-relativistic external shock. As mentioned previously, particle acceleration in the mildly relativistic regime remains open for study. On naive grounds, one may however expect these shocks to be very efficient particle accelerators for the following reasons: superluminality is no longer generic, just as shock compression of the magnetic field is less important; at a given magnetization, the precursor extends to larger distances, which opens the way for other instabilities, for instance resonant Whistler wave excitation (Lemoine \& Pelletier 2010), or MHD modes; electron heating to near equipartition remains envisageable, which would circumvent the problem of electron injection in non-relativistic shocks.

\subsubsection{Pulsar wind nebulae and active galactic nuclei}

The remarkable non-thermal spectrum of the Crab nebula indicates that electrons are accelerated to energies as high as a $\mathrm{PeV}$ at the termination shock of the relativistic pulsar wind, giving rise to synchrotron radiation up to $\mathrm{GeV}$ energies, see e.g Kirk et al. (2009) for a review. The changes in spectral slope indicate however that possibly two different mechanisms are at work: a hard powerlaw 
with $s \sim 1.5$ is observed up to TeV energies, and a Fermi-like powerlaw with $s \simeq 2.2$ is found above a $\mathrm{TeV}$, up to the $\mathrm{PeV}$.

The values of the Lorentz factor and the magnetization of the pulsar wind at the termination shock are not resolved. Actually, these quantities likely depend on the location on the termination shock surface, which is far from planar (Komissarov \& Lyubarsky 2003). However its is argued that $\gamma_{\mathrm{sh}} \gtrsim 100$ at least and $\sigma$ is not far below unity (Kirk et al. 2009). The model developed in the previous Section then indicates that Fermi acceleration should not be operative in such conditions. One possibility of course, is that wakefield acceleration of electrons and positrons occurs in the shock precursor if sufficiently many ions are present, as proposed by Hoshino et al. (1992), see however Kirk et al. (2009).

Given the rather nice agreement of the spectral slope expected for Fermi acceleration with that observed above a $\mathrm{TeV}$, one may turn the question otherwise and ask what would be needed to allow efficient Fermi acceleration to the highest energies. One interesting possibility is that shock driven reconnection of the striped wind leads to magnetic dissipation and electron/positron acceleration with the hard powerlaw index up to TeV (Lyubarsky 2003, Pétri \& Lyubarsky 2007, Sironi \& Spitkovsky 2011b). Results from particle-in-cell simulations are encouraging in this respect. How Fermi acceleration emerges at higher energies is not yet well understood, although one may speculate that the dissipation of magnetic field in the shock transition suffices to remove the phase space locking on magnetic field lines that was discussed earlier or, that reconnection accelerated electrons at a $\mathrm{TeV}$ - that carry most of the energy - propagate sufficiently far upstream to induce instabilities, that then provide a seed for scattering of higher energy particles.

Finally, in the case of blazars, Bl Lac objects or flat spectrum radio quasars, i.e. relativistic jets of active galactic nuclei that are beamed towards the observer, it is not known where the acceleration process takes place, whether in mildly relativistic internal shocks that are embedded in a relativistic flow, as for the prompt emission of gamma-ray bursts, or at the external shock for instance. In any case, recent inferences of hard powerlaws from blazar spectra at very high energies point to interesting acceleration physics in these objects (e.g. Ackermann et al. 2011).

\subsection{Acceleration to ultra-high energies}

A well known estimate of the maximal acceleration energy for non-relativistic shocks compares the acceleration timescale, $t_{\mathrm{acc}} \sim t_{\mathrm{scatt}} / \beta_{\mathrm{sh}}^{2}$ (with $t_{\mathrm{scatt}} \sim D / c^{2}$ the diffusion timescale in the shock environment) to the age of the shock wave $R /\left(\beta_{\mathrm{sh}} c\right)$ to infer (Lagage \& Césarsky 1983, Hillas 1984): $E_{\max } \sim \beta_{\mathrm{sh}} e R B$ if Bohm diffusion applies in the magnetic field $B$, meaning $t_{\mathrm{scatt}} \sim t_{\mathrm{L}}$. This estimate suggests that relativistic shock waves with $\beta_{\mathrm{sh}} \rightarrow 1$ appear as efficient particle accelerators to ultra-high energy.

However, if the scattering takes place in micro-turbulence, the scattering timescale increases quadratically with the energy $E$, since $t_{\text {scatt }} \sim t_{\mathrm{L}}^{2} c / \lambda_{B}$. In the presence of a mean magnetic field, one may argue that the upstream residence time is either governed by the scattering in the microturbulence, i.e. $t_{\mathrm{u}} \sim E^{2} /\left(\gamma_{\mathrm{sh}}^{2} e^{2} \delta B^{2} c \lambda_{B, \mathrm{u}}\right)$ or by the rotation of the particles in the background magnetic field $t_{\mathrm{u}} \sim E /\left(\gamma_{\mathrm{sh}} e B_{\mathrm{u}} c\right)$ (e.g. Pelletier et al. 2009). A Bohm estimate in the self-generated magnetic field is thus not supported by theory in the ultra-relativistic limit. In either limit above, ultra-relativistic shocks do not appear as promising accelerators to reach ultra-energies. For a 
quantitative estimate, consider the external shock of a gamma-ray burst at radius $R \sim 10^{17} \mathrm{~cm}$, assume that transport is governed by scattering in the upstream micro-turbulent field $\delta B_{\mid \mathrm{u}}$, assume that the acceleration timescale reduces to the residence time (a conservative bound), then the maximal electron energy, notwithstanding possible energy losses:

$$
E_{\max } \simeq \gamma_{\mathrm{sh}} \sqrt{R \lambda_{B}} Z e \delta B_{\mid \mathrm{u}} c \sim 10^{7} \mathrm{GeV} Z \epsilon_{B}^{1 / 2} R_{17} n_{\mathrm{u}, 0} .
$$

Mildly relativistic shocks appear as more promising sources of ultra-high energy particles. A reasonable guess is to expect a substantial magnetic amplification at such shocks with efficiency $\xi_{B} \sim 1-10 \%$, on rather large MHD scales. Such a configuration might possibly lead to a Bohm regime for particle transport. Then one would reach an optimal acceleration timescale with $t_{\text {acc }} \sim t_{\mathrm{L}}$ and maximal energy $E_{\max } \sim Z e \delta B$ (as written in the upstream rest frame).

To provide another perspective on this issue, one may express the acceleration timescale in units of the Larmor time in the total magnetic field, i.e. $t_{\mathrm{acc}}=\mathcal{A} t_{\mathrm{L}}$ and relate the maximal energy to the magnetic luminosity of the source (e.g. Norman et al. 1995, Waxman 2005, Lemoine \& Waxman 2009). Assuming that the acceleration site resides in a relativistic outflow with bulk Lorentz factor $\gamma$ (velocity $\beta c$ ), beamed towards the observer, with half-opening angle $\Theta$, limiting the maximal energy by the dynamical timescale as previously, one finds that the source frame magnetic luminosity of the source $L_{B}=R^{2} \Theta^{2} \gamma^{2} \beta c B^{2} / 4$ must verify

$$
L_{B} \geq 0.65 \times 10^{45} \Theta^{2} \gamma^{2} \mathcal{A}^{2} \beta^{3} Z^{-2} E_{20}^{2} \mathrm{erg} / \mathrm{s},
$$

in order to produce $10^{20} E_{20} \mathrm{eV}$ particles (of charge $Z$ ) in the observer rest frame. This bound generalizes the previous Lagage \& Césarsky (1983) limit to relativistic outflows. It is quite remarkable to note that only a handful of relativistic sources appear capable of accelerating particles to the most extreme energies recorded if $Z \sim 1$. In particular, as discussed in Lemoine \& Waxman (2009), this excludes standard active galactic nuclei or even blazars if the accelerated species are protons, while jets of FR-I radio-galaxies might possibly accelerate heavier particles to $Z \times 10^{18} \mathrm{eV}$. Whether the most extreme cosmic rays are protons or irons is a current subject of controversy, which clearly influences the debate on the nature of their sources. Such considerations lie however beyond the scope of this review.

\section{Conclusion}

The three facets of a collisionless shock structure, which include a reflecting barrier for a part of the incoming particles, the generation of supra-thermal particles and the generation of magnetic turbulence altogether frame a successful paradigm that applies to astrophysical shocks, both nonrelativistic and relativistic. Although, the phenomenology in the ultra-relativistic shock limit differs appreciably from that in the non-relativistic limit as the shock front then moves at a velocity close to $c$, about as fast as the accelerated particle. While in non-relativistic shocks, suprathermal particles isotropize and diffuse in the magnetized turbulence on both sides of the shock front, the suprathermal population around relativistic shocks remains highly anisotropic. In non-relativistic shocks, the small energy gain per cycle around the shock wave is compensated by a small escape probability 
through advection with the shocked plasma, while in relativistic shocks, this probability is high but the energy gain is also substantial. While the precursor of non-relativistic shocks extends to very large scales, that of relativistic shocks lies on scales intermediate between the plasma skin depth scale and the Larmor scale of the cycling particles. Finally, while perpendicular shock acceleration may be efficient at non-relativistic velocities, ultra-relativistic shocks are generically perpendicular and for them, acceleration is prohibited unless small scale turbulence has been excited in the shock vicinity.

The above picture has by now been understood analytically and tested, in part, by sophisticated particle-in-cell simulations. One thus understands why Fermi acceleration should not occur at ultra-relativistic shocks with magnetization of order unity, a rather frequent situation in high energy astrophysics: the larger the Lorentz factor, or the larger the magnetization, the shorter the precursor, hence the lesser the amount of time available for excitation of short scale turbulence by reflected particles. As we have argued here, the dividing line appears to be $\xi_{\mathrm{cr}}^{-1} \sigma \gamma_{\mathrm{sh}}^{2} \sim 1$ : for larger values of the l.h.s., growth of the filamentation instability and of other microturbulent modes, is suppressed.

Of course, additional effects may help acceleration proceed in a magnetized environment. Among these, one may cite the wakefield acceleration of electrons (for an $e-p$ shock) in precursor waves emitted by the incoming protons, the acceleration of particles in shock driven reconnection of an alternating upstream magnetic field, or radiation driven acceleration through repeated charged to neutral state conversions. And, of course, many questions remain open, among which: how are electrons heated in the shock transition and whether/how they reach equipartition with the protons, how do the electrons radiate and whether the radiative signature may provide a diagnosis of the turbulent state, whether the shock front remains stationary or whether it may suffer from corrugation or reformation, how acceleration proceeds at mildly relativistic shocks, etc.

Acknowledgments: We acknowledge support from the GDR-PCHE and the PEPS/PTI program of CNRS/INP.

\section{References}

[1] A. Achterberg, Y. Gallant, J. G. Kirk, A. W. Guthmann, A. W., MNRAS 328, 393-408 (2001)

[2] A. Achterberg, J. Wiersma, Astron. Astrophys., 475, 19-36 (2007)

[3] A. Achterberg, J. Wiersma, C. A. Norman, Astron. Astrophys., 475, 1-18 (2007)

[4] M. Ackermann et al. (Fermi Collaboration), arXiv:1108.1420 (2011)

[5] J. Bednarz, M. Ostrowski, Phys. Rev. Lett., 80, 3911-3914 (1998)

[6] M. C. Begelman, J. G. Kirk, ApJ, 353, 66-80 (1990)

[7] A. Bell, MNRAS, 182, 147-156 (1978) 
[8] R. Blandford, C. McKee, Phys. Fluids, 19, 1130-1138 (1976)

[9] A. Bret, M.-C. Firpo, C. Deutsch, Phys. Rev. Lett., 94, 115002 (2005a)

[10] A. Bret, M.-C. Firpo, C. Deutsch, Phys. Rev. E, 72, 016403 (2005b)

[11] A. Bret, ApJ, 699, 990-1003 (2009)

[12] A. Bret, L. Grémillet, D. Bénisti, Phys. Rev. E, 81, 036402 (2010)

[13] P. Chang, A. Spitkovsky, J. Arons, ApJ, 674, 378-387 (2008)

[14] E. V. Derishev, F. A. Aharonian, V. V. Kocharovsky, Vl. V., Kocharovsky, Phys. Rev. D, 68, 043003 (2003)

[15] D. Ellison, G. Double, Astropart. Phys., 18, 213-228 (2002)

[16] G. D. Fleishman, I. N. Toptygin, MNRAS, 381, 1473-1481 (2007).

[17] G. D. Fleishman, F. A. Urtiev, MNRAS, 406, 644-655 (2010).

[18] Y. A. Gallant, A. Achterberg, MNRAS, 305, L6-L10 (1999)

[19] Y. A. Gallant, M. Hoshino, A. B. Langdon, J. Arons, C. E. Max, ApJ, 391, 73-101 (1992)

[20] A. Gruzinov, E. Waxman, ApJ, 511, 852-861 (1999)

[21] A. M. Hillas, Ann. Rev. Astron. Astrophys., 22, 425-444 (1984).

[22] M. Hoshino, ApJ, 672, 940-956 (2008)

[23] M. Hoshino, J. Arons, Phys. Fluids B, 3, 818-833 (1991)

[24] M. Hoshino, J. Arons, Y. A. Gallant, A. B. Langdon, ApJ, 390, 454-479 (1992).

[25] B. Katz, U. Keshet, E. Waxman, ApJ, 655, 375-390 (2007)

[26] U. Keshet, Phys. Rev. Lett., 97, 221104 (2006)

[27] U. Keshet, B. Katz, A. Spitkovsky, E. Waxman, E., ApJ, 693, L127 (2009)

[28] U. Keshet, E. Waxman, Phys. Rev. Lett., 94, 111102 (2005)

[29] J. G. Kirk, A. W. Guthmann, Y. A. Gallant, A. Achterberg, ApJ, 542, 235-242 (2000)

[30] J. G. Kirk, Y. Lyubarsky, J. Pétri, in "Neutron stars and pulsars", Astrophysics and Space Science Library, 357, Springer, Berlin Heidelberg, 2009, p.421.

[31] J. G. Kirk, B. Reville, ApJ, 710, L16-L20 (2010)

[32] S. S. Komissarov, Y. E. Lyubarsky, MNRAS, 344, L93-L96 (2003) 
[33] P.-O. Lagage, C. Césarsky, Astron. Astrophys., 125, 249-257 (1983).

[34] M. Lemoine, G. Pelletier, ApJ, 589, L73-L76 (2003)

[35] M. Lemoine, G. Pelletier, MNRAS, 402, 321-334 (2010)

[36] M. Lemoine, G. Pelletier, MNRAS, 417, 1148-1161 (2011a)

[37] M. Lemoine, G. Pelletier, MNRAS, to appear, arXiv:1103.4823 (2011b)

[38] M. Lemoine, G. Pelletier, B. Revenu, ApJ, 645, L129-L132 (2006)

[39] M. Lemoine, B. Revenu, MNRAS, 366, 635-644 (2006)

[40] M. Lemoine, E. Waxman, 2009, JCAP, 11, 009

[41] M. M. Leroy, Phys. Fluids, 26, 2742-2753 (1983).

[42] Z. Li, E. Waxman, ApJ, 651, 328-332 (2006)

[43] Z. Li, X.-H. Zhao, JCAP, 05, 008 (2011)

[44] Y. Lyubarsky, MNRAS, 345, 153-160 (2003).

[45] Y. Lyubarsky, D. Eichler, ApJ, 647, L1250-L1254 (2006)

[46] S. F. Martins, R. A. Fonseca, L. O. Silva, W. B. Mori, ApJ, 695, L189-L193 (2009).

[47] M. V. Medvedev, ApJ, 540, 704-714 (2000)

[48] M. V. Medvedev, ApJ, 637, 869-872 (2006).

[49] M. V. Medvedev, A. Loeb, ApJ, 526, 697-706 (1999)

[50] M. Milosavljević, E. Nakar, ApJ, 651, 979-984 (2006)

[51] J. Niemiec, M. Ostrowski, ApJ, 641, 984-992 (2006)

[52] J. Niemec, M. Ostrowski, M. Pohl, ApJ, 650, 1020-1027 (2006)

[53] C. A. Norman, D. B. Melrose, A. Achterberg, ApJ, 454, 60-68 (1995).

[54] G. Pelletier, M. Lemoine, A. Marcowith, MNRAS, 393, 587-597 (2009)

[55] J. Pétri, Y. Lyubarsky, Astron. Astrophys., 473, 683-700 (2007)

[56] T. Piran, Rev. Mod. Phys., 76, 1143-1210 (2005)

[57] I. Rabinak, B. Katz, E. Waxman, ApJ, 736, 157-164 (2011)

[58] L. Sironi, A. Spitkovsky, ApJ, 698, 1523-1549 (2009) 
[59] L. Sironi, A. Spitkovsky, ApJ, 726, 75-99 (2011a)

[60] L. Sironi, A. Spitkovsky, arXiv:1107.0977v1 (2011b)

[61] A. Spitkovsky, ApJ 682, L5-L8 (2008)

[62] M. Vietri, ApJ, 591, 954-961 (2003).

[63] E. Waxman, Physica Scripta, T121, 147-152 (2005).

[64] J. Wiersma, A. Achterberg, Astron. Astrophys., 428, 365-371 (2004) 